\title{
Mass dependence of the surface $\delta$-interaction strength for two-particle (two-hole) multiplets
}

\author{
A. Heusler ${ }^{1, a}$ and P. von Brentano ${ }^{2}$ \\ 1 Max-Planck-Institut für Kernphysik, D-69029 Heidelberg, Germany \\ 2 Institut für Kernphysik, Universität zu Köln, D-50937 Köln, Germany
}

Received: 25 March 2008 / Revised: 28 July 2008

Published online: 16 September 2008 - (C) Società Italiana di Fisica / Springer-Verlag 2008

Communicated by R. Krücken

\begin{abstract}
By using the surface $\delta$-interaction, the lowest states in nuclei of atomic mass number $6 \leq A \leq$ 210 with a doubly magic core and two valence nucleons are investigated. A single parameter describes the strength of the multiplet splitting of the lowest configuration which includes the ground state. The dependence of the strength on the atomic mass number of the doubly magic core is determined as a power law function $A^{-f}$ with an exponent $f=\frac{2}{3}$ for $50 \lesssim A \leq 208$.
\end{abstract}

PACS. 21.30.Fe Forces in hadronic systems and effective interactions -21.60 . Cs Shell model $-27.60 .+\mathrm{j}$ $90 \leq A \leq 149-27.80$. +w $190 \leq A \leq 219$

\section{Introduction}

The nuclear shell model is the basic model in nuclear physics [1-10]. The shell model describes nuclei by valence protons and valence neutrons outside a semi- or doubly magic core.

In this paper we investigate the simplest nuclei for which the shell model can be applied [7-10]. These are the nuclei which have one or two particles (holes) outside a doubly magic core with mass numbers $A+\delta, A+2 \delta$, and $A$, respectively. Here the parameter $\delta=+1, \delta=-1$ refers to the systems with particles and holes, respectively.

We will use the surface $\delta$-interaction (SDI) which is a simple and yet rather successful theoretical description with only one parameter [11-17].

The SDI is presented extensively in several textbooks [5-10] and known to describe the complete groundstate multiplet in a two-particle (two-hole) system in various nuclei fairly well. Of course, more complicated systems and interactions have been studied [18-22]. Yet in this paper we consider only the SDI interaction for systems $A+2 \delta$.

Our goal is to find the global dependence of the multiplet splitting on the atomic mass number $A$, especially in medium and heavy nuclei. The global dependence described as the dependence of the effective strength of the surface $\delta$-interaction on the atomic mass number has not been studied in such a detail before. Presumably, because at the time when the SDI was introduced few experimental

\footnotetext{
a e-mail: A.Heusler@mpi-hd.mpg.de
}

data on nuclei $A+2 \delta$ near the doubly magic nuclei ${ }_{40}^{90} \mathrm{Zr}_{50}$, ${ }_{50}^{100} \mathrm{Sn}_{50},{ }_{50}^{132} \mathrm{Sn}_{82}$ were available. Only recently data near ${ }_{28}^{78} \mathrm{Ni}_{50}$ have become available [23].

As another method to derive the global dependence of the strength of the surface $\delta$-interaction on the atomic mass number, the differences between the masses of the nuclei with one and two valence nucleons and the mass of the doubly magic core can be investigated. However for nuclei $A, A+\delta, A+2 \delta$ in the single region of interest, $50 \lesssim A<208$, most mass differences are known with a precision of $10-100 \%$ only. In addition, the influence of the Coulomb force and the configuration mixing introduce systematic uncertainties up to $30 \%$.

\section{Multiplet splitting}

We investigate systems $A+2 \delta$ with a doubly magic core of atomic mass number $A=N+Z$ ( $N$ neutrons, $Z$ protons) and two valence nucleons $(\delta= \pm 1)$. In case the isospin of the core $T_{z}^{\text {core }}=(N-Z) / 2$ is zero or is large, $T_{z}^{\text {core }} \gg 1$ (see Chapt. 18 of ref. [8]), the wave function of a single configuration state is given by

$$
\begin{aligned}
& \Psi\left(A+2 \delta, j, j^{\prime}, t_{z}, t_{z}^{\prime}, J, T\right)= \\
& \left(\Psi^{\text {core }}\left(A, T^{\text {core }}\right) \otimes \Psi^{\text {nucl }}\left(j, j^{\prime}, t_{z}, t^{\prime}{ }_{z}, J, T\right)\right) .
\end{aligned}
$$

Here $j, j^{\prime}$ and $t_{z}, t^{\prime}{ }_{z}$ denote the spin and isospin of the two valence nucleons and $T$ the isospin of the two-nucleon subsystem, $J$ the total spin of the state. 
The mass of the corresponding state depends on the same quantum numbers, $A+2 \delta, j, j^{\prime}, t_{z}, t^{\prime}{ }_{z}, J, T$. In the following, $M$ will always refer to the mass of the system $A+2 \delta$ and we only consider the special case where the valence nucleons reside in the same orbit $j$. Further we assume the valence nucleons to be identical. However, in sect. 4.3 we briefly mention two odd-odd nuclei discussing the case $t_{z} \neq t_{z}^{\prime}$. Therefore the mass of the system $A+2 \delta$ is written as

$$
M\left(A, j, t_{z}, J, T\right) .
$$

We compare the multiplet splitting for the configuration containing the ground state only. The mass difference for the nucleus $A+2 \delta$ with total spin $J$ in reference to the mass with maximum spin $J_{\max }$ is thus given by

$$
\begin{aligned}
& \Delta M\left(A, j, t_{z}, J, T\right)= \\
& M\left(A, j, t_{z}, J, T\right)-M\left(A, j, t_{z}, J_{\max }, T\right) .
\end{aligned}
$$

The experimental multiplet splitting is derived from the excitation energies alone, taken from ENSDF [24], as

$$
\begin{aligned}
& \Delta M^{\operatorname{expt}}\left(A, j, t_{z}, J, T\right)= \\
& \frac{1}{c^{2}}\left[E_{x}^{\exp t}\left(A, j, t_{z}, J, T\right)-E_{x}^{\operatorname{expt}}\left(A, j, t_{z}, J_{\max }, T\right)\right] .
\end{aligned}
$$

Here $J_{\max }$ is chosen as the total spin $J$ of the state with the highest excitation energy. Especially, we have

$$
J_{\max }=2 j-1, \quad \text { for } T=1 .
$$

\subsection{Surface $\delta$-interaction}

The SDI was introduced [11] as a simple but yet successful method to calculate nuclear properties in the framework of the shell model. Following Chapts. $11,12^{1}$ of ref. [8] the interaction between two nucleons of zero range is assumed to be localized on the nuclear surface of the core. The interaction can be written as

$$
\begin{aligned}
\mathbf{V}_{12}\left(A, t_{z}\right)= & -\tilde{V}_{0}\left(A, t_{z}\right) \frac{\delta\left(r_{1}-r_{2}\right)}{r_{1} r_{2}} \\
& \times \delta\left(\cos \theta_{1}-\cos \theta_{2}\right) \delta\left(\phi_{1}-\phi_{2}\right)
\end{aligned}
$$

in spherical coordinates. The radial integral evaluated as

$$
C_{0}(n l)=\frac{1}{4 \pi} \int R_{n l}^{4}(r) \frac{1}{r^{2}} \mathrm{~d} r
$$

is assumed to be equal for all orbits $n l$ with main quantum number $n$ and orbital angular momentum $l\left(j=l \pm \frac{1}{2}\right)$. Here

$$
V_{0}\left(A, t_{z}\right)=\tilde{V}_{0}\left(A, t_{z}\right) C_{0}(n l)
$$

denotes the strength factor for each nucleus $A+2 \delta$.

\footnotetext{
1 Equations (7), (9), (11), (12), (13) correspond to eqs. (11.10), (11.16), (11.38), (11.11), (11.39) of ref. [8], respectively.
}

For a pure two-particle configuration one finds the multiplet splitting by

$$
\Delta M^{S D I}\left(A, j, t_{z}, J, T\right)=\frac{1}{2} V_{0}\left(A, t_{z}\right) \Delta S^{S D I}(j, J, T),
$$

where

$$
\Delta S^{S D I}(j, J, T)=S^{S D I}(j, J, T)-S^{S D I}\left(j, J_{\max }, T\right)
$$

denotes a geometrical factor, a number. The factor $\frac{1}{2}$ enters into eq. (9) since both valence nucleons occupy the same orbit $j$. The geometrical factor is evaluated by

$$
S^{S D I}(j, J, 1)=-(2 j+1)^{2}\left(\begin{array}{ccc}
j & j & J \\
\frac{1}{2} & -\frac{1}{2} & 0
\end{array}\right)^{2}
$$

for $T=1$ with the special value for total spin $J=0$

$$
S^{S D I}(j, 0,1)=-(2 j+1) \text {. }
$$

For $T=0$ the geometrical factor is evaluated by

$$
\begin{aligned}
& S^{S D I}(j, J, 0)= \\
& -(2 j+1)^{2}\left(\begin{array}{ccc}
j & j & J \\
\frac{1}{2} & -\frac{1}{2} & 0
\end{array}\right)^{2}\left(1+\frac{(2 j+1)^{2}}{J(J+1)}\right) .
\end{aligned}
$$

The seniority is a good quantum number for the SDI. For the same isospin $T$ and for $j \geq \frac{5}{2}$, the spacings between the $j^{2}$ levels and between the $j^{2 j-1}$ levels are the same (see p. 565 of ref. [8]). Hence the same formulæ (eqs. (9)-(13)) apply as well to two-particle systems $A+2 \delta, \delta=+1$ as to two-hole systems $A+2 \delta, \delta=-1$. An example is shown in fig. 3 for ${ }_{20}^{42} \mathrm{Ca}_{22}$ and ${ }_{20}^{46} \mathrm{Ca}_{26}$ where the multiplet splitting is identical for the $1 f_{7 / 2}{ }^{2}$ and $1 f_{7 / 2}{ }^{6}$ configurations.

In reality, because the multiplet splitting varies from one system $A+2 \delta$ to another, eq. (9) will not hold exactly. But one can always exactly reproduce the experimental splitting between the states with spins $J=0$ and $J=$ $J_{\max }$ by defining a suitable strength parameter

$$
V_{0}^{e x p t}\left(A, t_{z}\right)=\frac{2 \Delta M^{\operatorname{expt}}\left(A, j, t_{z}, 0,1\right)}{\Delta S^{S D I}(j, 0,1)} .
$$

\subsection{Example for the multiplet splitting}

As an example, in fig. 1 (left side) the level scheme is displayed for the nucleus ${ }^{210} \mathrm{Po}$ with two protons in the orbit $1 h_{9 / 2}$ and ${ }^{208} \mathrm{~Pb}$ as the doubly magic core. The multiplet splitting (eq. (4)) is shown on a keV scale, too.

The lowest states have spins $0^{+}, 2^{+}, 4^{+}, 6^{+}, 8^{+}$. They consist mainly of the configuration $h_{9 / 2}^{2}$ while the next multiplet is described by the configuration $1 h_{9 / 2} 2 f_{7 / 2}$, see table 3 . The energy difference between each pair of states with spins $2^{+}, 4^{+}, 6^{+}, 8^{+}$in these two multiplets is at least three times the mean splitting in each multiplet [24]. Therefore the admixture of other configurations to the ground-state multiplet can be assumed to be small. 


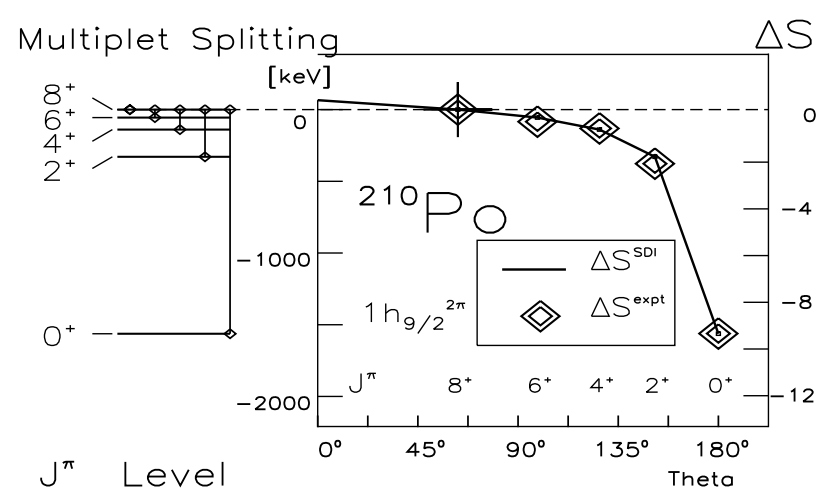

Fig. 1. Multiplet splitting for the lowest states in ${ }_{84}^{210} \mathrm{Po}_{126}$ with dominant configuration $h_{9 / 2}^{2 \pi}$. Left: level scheme for the ground-state multiplet with valence protons of $\operatorname{spin} j=\frac{9}{2}$ and total spins $J^{\pi}=0^{+}, 2^{+}, 4^{+}, 6^{+}, 8^{+}$derived from experimental excitation energies. The vertical lines show the multiplet splitting $\Delta M^{\text {expt }}$ (eq. (4)) between the states with spins $J=0,2,4,6,8$ and $J=J_{\max }=8$. Right: multiplet splitting $\Delta M^{\text {expt }}$ transformed to $\Delta S^{\text {expt }}$ (eq. (16), right scale) for $J^{\pi}=0^{+}, 2^{+}, 4^{+}, 6^{+}, 8^{+}$(diamonds) plotted versus the classical angle $\theta$ (eq. (15)). The cross and the dashed line mark the reference value $\Delta S^{\text {expt }}=0$ from which the multiplet splitting is calculated (eqs. (5), (16)). The drawn curve connects the calculated values $\Delta S^{S D I}$ (eq. (10)) in order to guide the eye. It is continued until $\Delta S^{\text {expt }}=-S^{S D I}\left(j, J_{\max }\right)=0.401$ at $\theta=0^{\circ}$.

We introduce the classical angle between the two valence nucleons as a convenient method to present the multiplet splitting. It is defined as (see eq. (II.2) in ref. [21])

$$
\begin{aligned}
& \theta\left(j, j^{\prime}, J\right)= \\
& \arccos \left(\frac{J(J+1)-j(j+1)-j^{\prime}\left(j^{\prime}+1\right)}{2 \sqrt{j(j+1) j^{\prime}\left(j^{\prime}+1\right)}}\right),
\end{aligned}
$$

where $j, j^{\prime}$ are the spins of the two interacting nucleons in a state with total spin $J$. In this paper we deal with $j=j^{\prime}$ only, see eq. (3).

The central part (diamonds in fig. 1) shows the experimental multiplet splitting $\Delta M^{\text {expt }}$ (eq. (4)) as function of the angle $\theta$ (eq. (15)). The reference energy for the state with spin $J_{\max }$ (eq. (5)) is marked by a cross. A parabolic dependence is found.

Similar to the calculated multiplet splitting (eq. (9)), the corresponding experimental values are given by

$$
\begin{aligned}
& \Delta S^{\operatorname{expt}}\left(A, j, t_{z}, J, T\right)=\frac{2 \Delta M^{\operatorname{expt}}\left(A, j, t_{z}, J, T\right)}{V_{0}^{\exp t}\left(A, t_{z}\right)}= \\
& \frac{\Delta M^{\operatorname{expt}}\left(A, j, t_{z}, J, T\right)}{\Delta M^{\operatorname{expt}}\left(A, j, t_{z}, 0,1\right)}\left(S^{S D I}\left(j, J_{\max }, 1\right)-(2 j+1)\right)
\end{aligned}
$$

by using eqs. (10), (12).

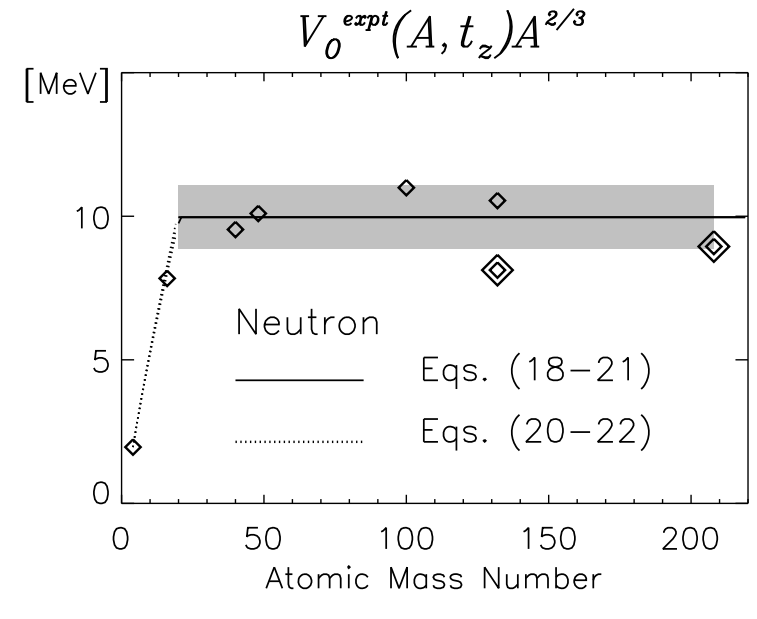

$V_{o}^{\exp t}\left(A, t_{z}\right) A^{2 / 3}$

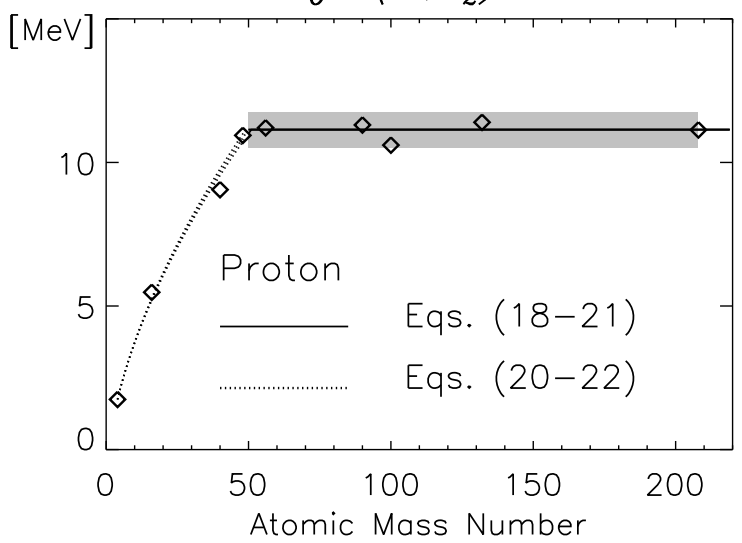

Fig. 2. Dependence of the experimental multiplet splitting between the ground state and the state with spin $J_{\max }$ (eq. (5)) on the atomic mass number $A$ of the core (eq. (1)). The shaded areas cover the values used to derive the mean strength $\bar{v}_{0}$ and its uncertainty (eq. (21)). For protons it is about 10\% larger than for neutrons. The exponent $f=\frac{2}{3}$ for the power law function $A^{-f}$ (eqs. (18), (19)) is valid only for heavier nuclei $A>A_{\min }$ (eq. (20), drawn line). The dotted curve shows the fit by interpolating (eq. (22)) between the experimental data point at $A=4$ and the flat region with an exponent $f=\frac{2}{3}$. Double diamonds mark values for multiplets where the main quantum number is $n=2\left({ }_{52}^{134} \mathrm{Sn}_{82}\right.$ and $\left.{ }_{82}^{210} \mathrm{~Pb}_{128}\right)$; otherwise it is $n=1$.

\section{Results}

\subsection{SDI strength parameter}

In order to find the strength $V_{0}$ (eq. (8)) we look at fig. 2, see also table 1 . Here we plot the quantity $V_{0}^{\text {expt }} A^{2 / 3}$ for the two-proton and two-neutron multiplets. One finds

$$
\begin{aligned}
& V_{0}^{\text {expt }}\left(A,+\frac{1}{2}\right) A^{2 / 3} \approx 10.3[\mathrm{MeV}] \text { for } A>20, \\
& V_{0}^{\text {expt }}\left(A,-\frac{1}{2}\right) A^{2 / 3} \approx 11.4[\mathrm{MeV}] \text { for } A>50 .
\end{aligned}
$$




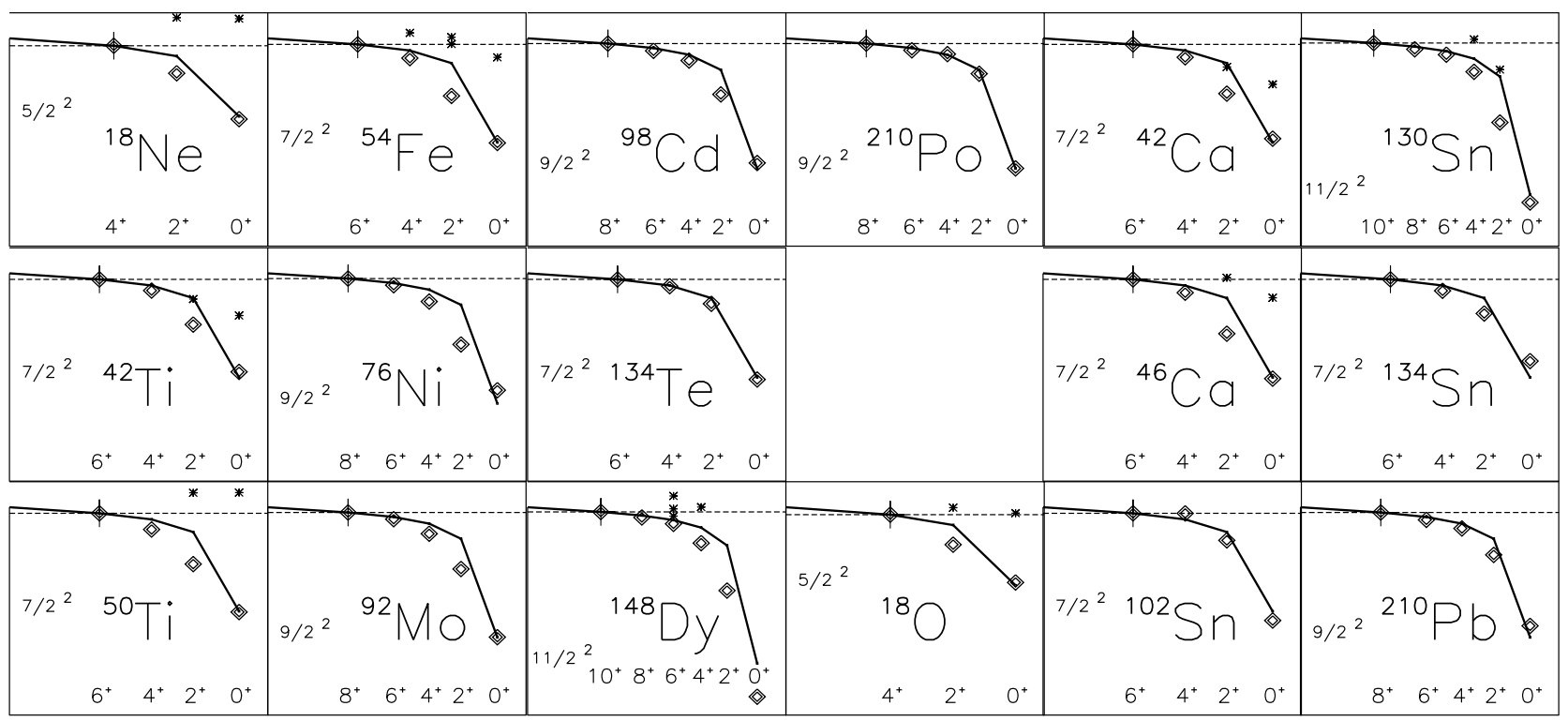

Fig. 3. Multiplet splitting of the lowest states in nuclei with a double magic core and two valence nucleons. For a detailed description, see fig. 4. First the nuclei with valence protons are shown until the empty frame by reading from the upper left column-wise downwards, thereafter the nuclei with valence neutrons are shown. Some higher excited states are shown by asterisks.

For heavier nuclei, this suggests the fit formula by a power law function

$$
V_{0}^{f i t}\left(A, t_{z}\right)=\bar{v}_{0}\left(t_{z}\right) A^{-f\left(A, t_{z}\right)}
$$

with an index

$$
\begin{aligned}
& f=\frac{2}{3}, \quad \text { for } A>A_{\min }\left(t_{z}\right) \\
& A_{\min }\left(+\frac{1}{2}\right)=20, \quad A_{\min }\left(-\frac{1}{2}\right)=50 \\
& \text { (neutron) } \quad \bar{v}_{0}\left(+\frac{1}{2}\right)=10.3 \pm 1.4[\mathrm{MeV}] \\
& \text { (proton) } \quad \bar{v}_{0}\left(-\frac{1}{2}\right)=11.4 \pm 0.9[\mathrm{MeV}]
\end{aligned}
$$

For lighter nuclei the fit formula is more complicated. By interpolating linearly between $A=4$ and $A_{\text {min }}$ on a doubly logarithmic scale one obtains for $4 \leq A \leq A_{\min }\left(t_{z}\right)$

$$
V_{0}^{f i t}\left(A, t_{z}\right)=\bar{v}_{0}\left(t_{z}\right) A^{-2 / 3}\left(\frac{A}{A_{\min }\left(t_{z}\right)}\right)^{-\delta f\left(t_{z}\right)}
$$

with

$$
\delta f\left(t_{z}\right)=\log \left(\frac{V_{0}^{\text {expt }}\left(4, t_{z}\right) 4^{2 / 3}}{\bar{v}_{0}\left(t_{z}\right)}\right) / \log \left(\frac{4}{A_{\min }\left(t_{z}\right)}\right) .
$$

The origin for the deviation of the fit function from $A^{-2 / 3}$

\begin{tabular}{|c|c|c|c|c|c|c|}
\hline Nucleus & Core & $n$ & $l$ & $j$ & $\begin{array}{c}V_{0}^{\text {expt }} A^{2 / 3} \\
\text { Eq. (14) } \\
{[\mathrm{MeV}]} \\
\end{array}$ & $\begin{array}{c}V_{0}^{\text {fit }} A^{2 / 3} \\
\text { Eq. (18) } \\
{[\mathrm{MeV}]}\end{array}$ \\
\hline${ }_{42}^{92} \mathrm{Mo}_{50}$ & ${ }_{40}^{90} \mathrm{Zr}_{50}$ & 1 & 4 & $9 / 2$ & 11.6 & 11.4 \\
\hline${ }_{48}^{98} \mathrm{Cd}_{50}$ & ${ }_{50}^{100} \mathrm{Sn}_{50}$ & 1 & 4 & $9 / 2$ & 10.9 & 11.4 \\
\hline${ }_{50}^{102} \mathrm{Sn}_{52}$ & ${ }_{50}^{100} \mathrm{Sn}_{50}$ & 1 & 4 & $7 / 2$ & 11.3 & 10.3 \\
\hline${ }_{50}^{130} \mathrm{Sn}_{80}$ & ${ }_{50}^{132} \mathrm{Sn}_{82}$ & 1 & 5 & $11 / 2$ & 10.8 & 10.3 \\
\hline${ }_{50}^{134} \mathrm{Sn}_{84}$ & ${ }_{50}^{132} \mathrm{Sn}_{82}$ & 2 & 3 & $7 / 2$ & 8.6 & 10.3 \\
\hline${ }_{52}^{134} \mathrm{Te}_{82}$ & ${ }_{50}^{132} \mathrm{Sn}_{82}$ & 1 & 4 & $7 / 2$ & 11.6 & 11.4 \\
\hline${ }_{82}^{210} \mathrm{~Pb}_{128}$ & ${ }_{82}^{208} \mathrm{~Pb}_{126}$ & 2 & 4 & $9 / 2$ & 9.3 & 10.3 \\
\hline${ }_{84}^{210} \mathrm{Po}_{126}$ & ${ }_{82}^{208} \mathrm{~Pb}_{126}$ & 1 & 5 & $9 / 2$ & 11.4 & 11.4 \\
\hline
\end{tabular}
for lighter nuclei is not easy to explain.
Table 1. The interaction strengths $V_{0}^{\text {expt }}$ (eq. (14)) for heavy nuclei $A+2 \delta$ with a core of atomic mass number $A \geq 90$. The quantum numbers $n l j$ of the valence nucleons are given. By averaging the values for $n=1$ only one finds $V_{0}^{f i t} A^{2 / 3}=$ $11.3 \pm 0.5$.

\subsection{Multiplet splitting for the ground state}

Equation (14) is valid only for pure configuration states. In deriving the strength factor $V_{0}^{f i t}$ (eq. (18)) we select states which can be assumed to be rather pure.

The multiplet splitting is largest for the $0^{+}$ground state (eq. (12)). Therefore we consider only configurations where the valence nucleons occupy orbits with spins $j \geq \frac{5}{2}$. In most nuclei the excitation energy of the lowest $2^{+}$state is lower than predicted by the SDI because several configurations admix. As seen in fig. 3, only for ${ }^{134} \mathrm{Te}$ and ${ }^{210} \mathrm{Po}$ the multiplet splitting is very well described by the SDI. 


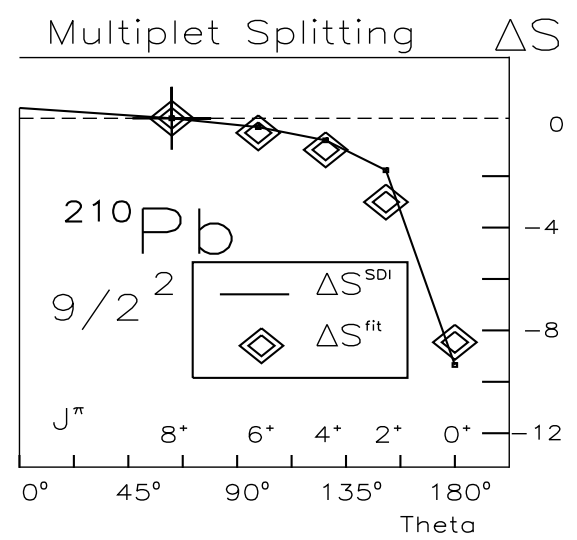

Fig. 4. Multiplet splitting for the ground-state multiplet $2 g_{9 / 2}{ }^{2 \nu}$ in ${ }_{84}^{210} \mathrm{~Pb}_{126}$. The diamonds show the values $\Delta S^{f i t}$ (eq. (25)), the drawn line the values $\Delta S^{S D I}$ (eq. (10)).

Similarly, the excitation energies for states with higher spins $2<J<J_{\max }$ are often lowered by admixing configurations.

Besides the ground state, only the multiplet member with the highest spin $J_{\max }$ is rather pure in most nuclei with a doubly magic core. For this reason we discuss only the multiplet splitting between the states with spins $J=0$ and $J=J_{\max }$ (eq. (5)).

For ${ }^{148}$ Dy the ground state is lower than calculated whereas the other multiplet members are fairly well described. A large admixture of another configuration to the ground state may be the reason, see sect. 4.1.2. Similarly, higher values for the ground state in ${ }^{134} \mathrm{Sn}$ and ${ }^{210} \mathrm{~Pb}$ are discussed in sect. 4.2.

Corresponding to eq. (9) we define

$$
\begin{aligned}
& \Delta M^{f i t}\left(A, j, t_{z}, J, T\right)= \\
& \frac{1}{2} V_{0}^{f i t}\left(A, t_{z}\right) \Delta S^{S D I}(j, J, T) .
\end{aligned}
$$

The ratio between the experimental and the calculated multiplet splitting for the ground state (eqs. (4), (23))

$$
R\left(A, j, t_{z}\right)=\frac{\Delta M^{\operatorname{expt}}\left(A, j, t_{z}, 0,1\right)}{\Delta M^{f i t}\left(A, j, t_{z}, 0,1\right)}
$$

is unity if the SDI describes the experimental multiplet splitting.

In table 3 the experimental values $\Delta M^{\text {expt }}$ (eq. (4)), the values $\Delta M^{\text {fit }}$ (eqs. (11), (18), (23)) calculated with the strength factor $\bar{v}_{0}$ (eq. (21)) and the function $A^{-f}$ (eqs. (18)-(22)) together with the ratio $R$ (eq. (24)) are listed for the ground state $(J=0)$.

\subsection{Multiplet splitting for all spins}

It is convenient to transform the experimental multiplet splitting into a number similar to $\Delta S^{S D I}$ (eq. (10)) by

$$
\begin{aligned}
& \Delta S^{f i t}\left(A, j, t_{z}, J, T\right)=\frac{2 \Delta M^{\operatorname{expt}}\left(A, j, t_{z}, J, T\right)}{V_{0}^{f i t}\left(A, t_{z}\right)}= \\
& \frac{\Delta M^{\operatorname{expt}}\left(A, j, t_{z}, J, T\right)}{\Delta M^{f i t}\left(A, j, t_{z}, J, T\right)} \Delta S^{S D I}(j, J, T) .
\end{aligned}
$$

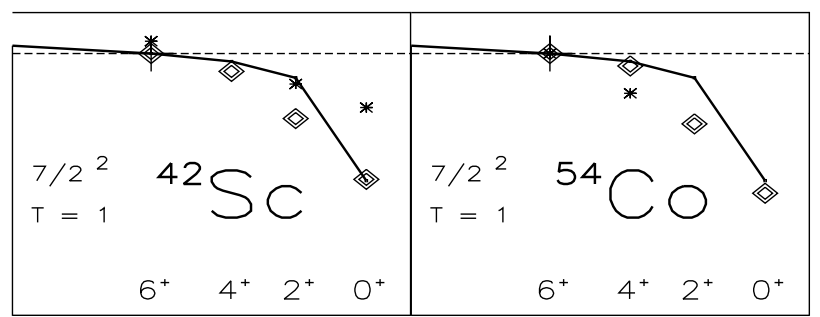

Fig. 5. Multiplet splitting for the $T=1$ states in the odd-odd nuclei ${ }^{42} \mathrm{Sc},{ }^{54} \mathrm{Co}$. For a detailed description, see fig. 4 . Some higher excited states are shown by asterisks.

For ${ }^{210} \mathrm{~Pb}$ as an example, fig. 4 shows the fitted multiplet splitting $\Delta S^{f i t}$ together with the values $\Delta S^{S D I}$. Figures 3,5 show the same values for nearly 20 nuclei with valence nucleons in different orbits $j$. While in fig. 4 a full description of all items is given, for clarity in figs. 3, 5 much of the description is omitted.

In contrast to ${ }^{210} \mathrm{Po}$ and ${ }^{134} \mathrm{Te}$ where $\Delta S^{\exp t}$ equals $\Delta S^{f i t}$ (eqs. (16), (25)) within a few percent for all spins $J$, for nuclei $A+2 \delta$ with valence nucleons of a certain spin $j$, the maximal experimental multiplet splitting is generally different from the fit value, $\Delta S^{\operatorname{expt}}\left(A, j, t_{z}, 0,1\right) \neq$ $\Delta S^{f i t}\left(A, j, t_{z}, 0,1\right)$.

Some higher excited states are indicated by asterisks (as far as spins are known), arbitrarily using the angle $\theta\left(j, j^{\prime}, J\right)$ (eq. (15)) with the same spins $j=j^{\prime}$ and $J$ as for the ground-state multiplet, since often the dominant configuration is not known (see table 3 ).

\section{Discussion}

\subsection{Multiplet splitting in even-even nuclei}

Figure 3 shows the multiplet splitting among about 60 states in nearly 20 nuclei with a doubly magic core $16 \leq$ $A \leq 208$.

For the nuclei ${ }^{18} \mathrm{O},{ }^{134} \mathrm{Te},{ }^{210} \mathrm{Po}$, ref. [22] studied the low-lying states with a more sophisticated interaction and more configurations. However, the SDI interaction (eqs. (18)-(21)) reproduces the experimental splitting for all members of the ground-state multiplet in ${ }^{134} \mathrm{Te}$ and ${ }^{210}$ Po equally well as already stated by ref. [13] (where the $8^{+}$states in ${ }^{210} \mathrm{Po}$ and ${ }^{210} \mathrm{~Pb}$ were not yet detected, however).

\subsubsection{Nuclei with a ${ }_{20}^{40} \mathrm{Ca}_{20},{ }_{20}^{48} \mathrm{Ca}_{28},{ }_{28}^{56} \mathrm{Ni}_{28}$ or ${ }_{28}^{78} \mathrm{Ni}_{50}$ core}

For ${ }_{20}^{46} \mathrm{Ca}_{26}$ and ${ }_{26}^{54} \mathrm{Fe}_{28}$, in the shell model the next configurations $s_{1 / 2}^{2}, d_{3 / 2}^{2}$ may admix to the $0^{+}, 2^{+}$states, but are expected at much higher excitation energies, while for ${ }_{20}^{42} \mathrm{Ca}_{22},{ }_{22}^{42} \mathrm{Ti}_{20},{ }_{22}^{50} \mathrm{Ti}_{28}$ the next configuration $f_{7 / 2} p_{3 / 2}$ may admix to the $2^{+}, 4^{+}$states only.

We note that for ${ }_{22}^{50} \mathrm{Ti}_{28}$ the low isospin of the core $\left(T_{z}^{c o r e}=4\right)$ may add another component to the wave function (eq. (1)). 
Table 2. The lowest states in ${ }^{42} \mathrm{Sc}[24,25],{ }^{54} \mathrm{Co}[24]$. For details see table 3.

\begin{tabular}{|c|c|c|c|c|c|}
\hline \multirow[t]{3}{*}{ Nucleus } & \multirow[t]{3}{*}{ Range of $J^{\pi}$} & \multirow[t]{3}{*}{ Main configuration } & \multicolumn{2}{|c|}{$\Delta M$} & \multirow[t]{2}{*}{$R$} \\
\hline & & & Expt. & Fit $^{1}$ & \\
\hline & & & $\begin{array}{c}\text { Eq. (4) } \\
{[\mathrm{keV}]}\end{array}$ & $\begin{array}{c}\text { Eq. (23) } \\
{[\mathrm{keV}]}\end{array}$ & Eq. (24) \\
\hline \multirow[t]{2}{*}{${ }_{21}^{42} \mathrm{Sc}_{21}$} & $0^{+}, 2^{+}, 4^{+}, \underline{6^{+}}$ & $\begin{array}{c}1 f_{7 / 2}^{\nu} 1 f_{7 / 2}^{\pi} \\
(T=1)\end{array}$ & -3281 & -3317 & 0.99 \\
\hline & $1^{+}, 3^{+}, \underline{5^{+}}, 7^{+}$ & $\begin{array}{c}1 f_{7 / 2}^{\nu} 1 f_{7 / 2}^{\pi} \\
(T=0)\end{array}$ & & & \\
\hline \multirow[t]{2}{*}{${ }_{27}^{54} \mathrm{Co}_{27}$} & $0^{+}, 2^{+}, 4^{+}, \underline{6^{+}}$ & $\begin{array}{c}1 f_{7 / 2}^{-\nu} 1 f_{7 / 2}^{-\pi} \\
(T=1)\end{array}$ & -2911 & -2651 & 1.10 \\
\hline & $1^{+}, 3^{+}, \underline{5^{+}}, 7^{+}$ & $\begin{array}{c}1 f_{7 / 2}^{-\nu} 1 f_{7 / 2}^{-\pi} \\
(T=0)\end{array}$ & & & \\
\hline
\end{tabular}

${ }^{1} v_{0}\left(t_{z}\right)$ with $t_{z}=+\frac{1}{2}$ has been used (eq. (21)).

The lowest multiplet of ${ }_{28}^{76} \mathrm{Ni}_{48}$ has been recently determined with tentative spin assignments $0^{+}, 2^{+}, 4^{+}, 6^{+}$, $8^{+}$[23]. The data are omitted in fig. 2 but shown in fig. 3.

\subsubsection{Nuclei with a ${ }_{40}^{90} \mathrm{Zr}_{50},{ }_{50}^{100} \mathrm{Sn}_{50},{ }_{50}^{132} \mathrm{Sn}_{82}$ or ${ }_{64}^{146} \mathrm{Gd}_{82}$ core}

In the nucleus ${ }_{42}^{92} \mathrm{Mo}_{50}$ the ground-state multiplet mainly consists of the configuration $g_{9 / 2}^{2}$. The multiplet splitting is well described by the SDI (fig. 3). We note, however, that the low isospin of the core $\left(T_{z}^{c o r e}=5\right)$ may add another component to the wave function (eq. (1)).

By using the tentative spin assignments [24] and by assuming the configuration $g_{9 / 2}^{2}$, a calculation reproduces the multiplet splitting for ${ }_{50}^{102} \mathrm{Sn}_{52}$ (fig. 3). Yet the energy of a second $0^{+}$state is uncertain [24].

The ground-state multiplet of ${ }_{66}^{148} \mathrm{Dy}_{82}$ is expected to have a dominant configuration $h_{9 / 2}^{2}$. In ${ }_{65}^{147} \mathrm{~Tb}_{82}$ however, the configuration $h_{11 / 2}$ is just $50 \mathrm{keV}$ above the configuration $s_{1 / 2}$ followed by $d_{3 / 2}$ lying $253 \mathrm{keV}$ higher in energy; in ${ }_{67}^{149} \mathrm{Ho}_{82}$ the configuration $h_{11 / 2}$ is just $49 \mathrm{keV}$ below the configuration $s_{1 / 2}$ followed by $d_{3 / 2}$ which is $171 \mathrm{keV}$ apart. In ${ }_{66}^{148} \mathrm{Dy}_{82}$ therefore, the lowest $0^{+}$and $2^{+}$states are expected to contain considerable admixtures of the configurations $s_{1 / 2}^{2}, s_{1 / 2} d_{3 / 2}, d_{3 / 2}^{2}$. The excitation energies of these two states being lower than predicted may thus be explained. The next states with spins $0^{+}$and $2^{+}$ (and $1^{+}$) expected to have low excitation energies are not yet known. Hence, the data are omitted in fig. 2 but shown in fig. 3.

\subsubsection{Nuclei with a ${ }^{208} \mathrm{~Pb}$ core}

In the nuclei ${ }^{210} \mathrm{~Pb}$ and ${ }^{210} \mathrm{Po}$ the configurations $g_{9 / 2}$ and $h_{9 / 2}$, respectively, produce states with spins $0^{+}, 2^{+}, 4^{+}$, $6^{+}, 8^{+}$, all of which can be expected to be rather pure. In the shell model the next configurations are at a large distance relative to the mean matrix element of the residual interaction of about $100 \mathrm{keV}$.

\subsection{Radial dependence}

A basic assumption of the SDI is the neglect of any dependence of the radial wave function on the details of the orbit (eq. (7)). In effect, the region where the valence nucleons interact is assumed to be similar for all nuclei (see figs. 3-4 of ref. [4]).

In tables 1,3 the quantum numbers $n l j$ of the orbits for the valence nucleons are given. In fig. 2, for protons as valence nucleons no significant dependence on the quantum numbers $n l j$ is visible; the main quantum number is $n=1$ throughout.

However, some dependence on the radial part of the wave function $C_{0}(n l)$ (eq. (7)) is indicated for nuclei with a doubly magic core ${ }_{50}^{132} \mathrm{Sn}_{82}$ and ${ }_{82}^{208} \mathrm{~Pb}_{126}$ where the valence neutrons have main quantum number $n=2$. Both values are lower than the mean value. By using only experimental values with main quantum number $n=1$ (single diamonds in fig. 2) the mean strength factor $\bar{v}_{0}$ (eq. (18)) is found to be the same for neutrons and protons for nuclei with a core of mass number $A \geq 90$ (table 1$)$.

\subsection{Odd-odd nuclei}

Only few odd-odd nuclei with a doubly magic core and two valence nucleons are known where the configuration mixing is assumed to be small, namely ${ }_{21}^{42} \mathrm{Sc}_{21}[25],{ }_{27}^{54} \mathrm{Co}_{27}$. Here, because the isospin of the valence nucleons differs, states with spins $J^{\pi}=1^{+}-7^{+}$are present (eq. (13)), see also table 2 .

For the states with isospin $T=0$ the values $\Delta S^{\operatorname{expt}}$ for the multiplet splitting are small and range near unity. For the states with isospin $T=1$, fig. 5 shows the multiplet splitting. Here the multiplet splitting is similar to that for the nuclei ${ }_{22}^{42} \mathrm{Ti}_{20},{ }_{20}^{42} \mathrm{Ca}_{22},{ }_{20}^{46} \mathrm{Ca}_{26},{ }_{22}^{50} \mathrm{Ti}_{28},{ }_{22}^{54} \mathrm{Fe}_{28},{ }_{50}^{102} \mathrm{Sn}_{52}$, ${ }_{52}^{134} \mathrm{Te}_{52},{ }_{50}^{134} \mathrm{Sn}_{84}$, which all have valence nucleons with spin $j=\frac{7}{2}$, see tables 2,3 and figs. 3,5 .

Since the exponent $f$ in the power law function (eq. (18)) starts to deviate from $f=\frac{2}{3}$ for lighter nuclei with mass number $A \lesssim 50$ (see fig. 2), we do not discuss the data from these odd-odd nuclei. 
Table 3. The lowest states in nuclei with a doubly magic core and two valence nucleons assumed to have a negligible admixture to some main configuration. The next configurations expected from the shell model to admix considerably are indicated. The order of the single-particle levels in dependence on the atomic mass number is taken from ref. [4]. The multiplet splitting between the state with the highest known spin $J_{\max }$ (eq. (5), underlined in the table) and the lowest spin (the ground state with spin $0^{+}$) is given by $\Delta M^{\text {expt }}$ (eq. (4)). By using the mean value $\bar{v}_{0}$ (eq. (21)) and the function $A^{-f}$ (eqs. (18)-(22)) the multiplet splitting is calculated as $\Delta M^{f i t}$ (eq. (23)). The ratio $R$ (eq. (24)) should be unity if the SDI describes the experimental multiplet splitting.

\begin{tabular}{|c|c|c|c|c|c|c|}
\hline \multirow[t]{3}{*}{ Nucleus } & \multirow[t]{3}{*}{ Range of $J^{\pi}$} & \multicolumn{2}{|c|}{ Configuration } & \multicolumn{2}{|c|}{$\Delta M$} & \multirow[t]{2}{*}{$R$} \\
\hline & & Main & Adm. & Expt. & Fit & \\
\hline & & & & $\begin{array}{c}\text { Eq. (4) } \\
{[\mathrm{keV}]}\end{array}$ & $\begin{array}{c}\text { Eq. (23) } \\
{[\mathrm{keV}]}\end{array}$ & Eq. (24) \\
\hline${ }_{2}^{6} \mathrm{He}_{4}$ & $0^{+}, \underline{2}^{+}$ & $1 d_{3 / 2}^{2 \nu}$ & & -1797 & 1 & $(1.0)$ \\
\hline${ }_{2}^{6} \mathrm{Be}_{4}$ & $0^{+}, \underline{2^{+}}$ & $1 d_{3 / 2}^{2 \pi}$ & & -1670 & 1 & (1.0) \\
\hline${ }_{8}^{18} \mathrm{O}_{10}$ & $\begin{array}{c}0^{+}, 2^{+}, \underline{4^{+}} \\
2^{+}, 3^{+}\end{array}$ & $1 d_{5 / 2}^{2 \nu}$ & $d_{5 / 2} s_{1 / 2}$ & -3554 & -3704 & 0.96 \\
\hline${ }_{10}^{18} \mathrm{Ne}_{8}$ & $\begin{array}{c}0^{+}, 2^{+}, \underline{4^{+}} \\
2^{+}, 3^{+}\end{array}$ & $1 d_{5 / 2}^{2 \pi}$ & $d_{5 / 2} s_{1 / 2}$ & -2613 & -2517 & 1.04 \\
\hline${ }_{20}^{42} \mathrm{Ca}_{22}$ & $\begin{array}{l}0^{+} \cdots \underline{6^{+}} \\
2^{+}-5^{+}\end{array}$ & $1 f_{7 / 2}^{2 \nu}$ & $f_{7 / 2} p_{3 / 2}$ & -3189 & -3317 & 0.96 \\
\hline${ }_{22}^{42} \mathrm{Ti}_{20}$ & $\begin{array}{l}0^{+} \cdots \underline{6^{+}} \\
2^{+}-5^{+}\end{array}$ & $1 f_{7 / 2}^{2 \pi}$ & $f_{7 / 2} p_{3 / 2}$ & -3043 & -3225 & 0.94 \\
\hline${ }_{20}^{46} \mathrm{Ca}_{26}$ & $\begin{array}{c}0^{+} \cdots \underline{6^{+}} \\
0^{+}, 2^{+}\end{array}$ & $1 f_{7 / 2}^{-2 \nu}$ & $d_{3 / 2}^{2}$ & -2973 & -2938 & 1.01 \\
\hline${ }_{22}^{50} \mathrm{Ti}_{28}$ & $\begin{array}{l}0^{+} \cdots \underline{6^{+}} \\
2^{+}-5^{+}\end{array}$ & $1 f_{7 / 2}^{2 \pi}$ & $f_{7 / 2} p_{3 / 2}$ & -3198 & -3175 & 1.01 \\
\hline${ }_{26}^{54} \mathrm{Fe}_{28}$ & $\begin{array}{c}0^{+} \cdots \underline{6^{+}} \\
0^{+}, 2^{+}\end{array}$ & $1 f_{7 / 2}^{-2 \pi}$ & $d_{3 / 2}^{2}$ & -2949 & -2934 & 1.00 \\
\hline${ }_{28}^{76} \mathrm{Ni}_{48}$ & $0^{+} \ldots \underline{6^{+}}$ & $1 g_{9 / 2}^{-2 \nu}$ & $p_{1 / 2}^{2}$ & $-2420^{2}$ & -2996 & 0.81 \\
\hline${ }_{42}^{92}$ Mo50 & $\begin{array}{l}0^{+} \cdots \underline{8^{+}} \\
2^{+}-7^{+}\end{array}$ & $1 g_{9 / 2}^{2 \pi}$ & $d_{5 / 2} g_{9 / 2}$ & -2760 & -2764 & 1.00 \\
\hline${ }_{48}^{98} \mathrm{Cd}_{50}$ & $0_{0^{+}} \ldots \underline{8^{+}}$ & $1 g_{9 / 2}^{-2 \pi}$ & $p_{1 / 2}^{2}$ & -2427 & -2539 & 0.96 \\
\hline${ }_{50}^{102} \mathrm{Sn}_{52}$ & $\begin{array}{l}0^{+} \cdots \underline{6^{+}} \\
1^{+}-6^{+}\end{array}$ & $1 g_{7 / 2}^{2 \nu}$ & $d_{5 / 2} g_{7 / 2}$ & -1969 & -1800 & 1.09 \\
\hline${ }_{50}^{130} \mathrm{Sn}_{80}$ & $\begin{array}{c}0^{+} \cdots \underline{10^{+}} \\
0^{+}, 2^{+}\end{array}$ & $1 h_{11 / 2}^{-2 \nu}$ & $d_{3 / 2}^{2}$ & -2434 & -2312 & 1.05 \\
\hline${ }_{50}^{134} \mathrm{Sn}_{84}$ & $\begin{array}{l}0^{+} \cdots \underline{6^{+}} \\
2^{+}-5^{+} \\
1^{+}-8^{+}\end{array}$ & $2 f_{7 / 2}^{2 \nu}$ & $\begin{array}{l}f_{7 / 2} p_{3 / 2} \\
f_{7 / 2} h_{9 / 2}\end{array}$ & -1247 & -1497 & 0.83 \\
\hline${ }_{52}^{134} \mathrm{Te}_{82}$ & $\begin{array}{l}0^{+} \cdots \underline{6^{+}} \\
1^{+}-6^{+}\end{array}$ & $1 g_{7 / 2}^{2 \pi}$ & $d_{5 / 2} g_{7 / 2}$ & -1691 & -1656 & 1.02 \\
\hline${ }_{66}^{148} \mathrm{Dy}_{82}$ & $\begin{array}{c}0^{+} \ldots \underline{10^{+}} \\
0^{+} \\
1^{+}, 2^{+}\end{array}$ & $1 h_{11 / 2}^{2 \pi}$ & $\begin{array}{c}s_{1 / 2}^{2} \\
s_{1 / 2} d_{3 / 2}\end{array}$ & -2919 & -2393 & 1.22 \\
\hline${ }_{82}^{210} \mathrm{~Pb}_{128}$ & $\begin{array}{l}0^{+} \cdots \underline{8^{+}} \\
1^{+}-10^{+}\end{array}$ & $2 g_{9 / 2}^{2 \nu}$ & $g_{9 / 2} i_{11 / 2}$ & -1278 & -1408 & 0.91 \\
\hline${ }_{84}^{210} \mathrm{Po}_{126}$ & $\begin{array}{l}0^{+} \cdots \underline{8^{+}} \\
1^{+}-8^{+}\end{array}$ & $1 h_{9 / 2}^{2 \pi}$ & $h_{9 / 2} f_{7 / 2}$ & -1557 & -1558 & 1.00 \\
\hline
\end{tabular}

1 The experimental values for the nuclei with a ${ }^{4} \mathrm{He}$ core are used for the fit of the function $A^{-f}$ (eq. (22)).

2 From ref. [23]. 


\section{Summary}

For nuclear systems $A+2 \delta$ with two valence nucleons outside a doubly magic core $A(\delta=+1, \delta=-1$ referring to particles and holes, respectively) the lowest multiplet built with the single particle (hole) in the ground state of the system $A+\delta$ is investigated. From the multiplet splitting of nearly 20 such systems, the strength of the residual interaction is found to be proportional to the atomic mass number by a power law function $A^{-f}$ with an exponent $f=\frac{2}{3}$ for atomic mass number $50 \lesssim A \leq 208$.

For light and medium heavy nuclei $(A<40)$ a departure of the power law function with an exponent $f=\frac{2}{3}$ is determined.

We thank J. Jolie, R.V. Jolos, A.F. Lisetskiy, K.H. Maier and C. Scholl for discussions. This work has been supported by Br799/13-1.

\section{References}

1. J.H.D. Jensen, M. Goeppert Mayer, Elementary Theory of Nuclear Shell Structure (Wiley, New York, 1955).

2. A. de-Shalit, I. Talmi, Nuclear Shell Theory (Academic Press, New York, 1963).

3. H. Feshbach, Nuclear Spectroscopy, edited by F. Ajzenberg Selove (Academic Press, New York, 1967).

4. A. Bohr, B.R. Mottelson, Nuclear Structure, Vol. I (W. A. Benjamin, New York, 1969).

5. P.J. Brussaard, P.W.M. Glaudemans, Shell Model Applications in Nuclear Spectroscopy (North-Holland, Amsterdam, 1977).

6. P. Ring, P. Schuck, The Nuclear Many Body Problem (Springer Verlag, Heidelberg, 1980).

7. R.F.A. Casten, Nuclear Structure From A Simple Perspective (Oxford University Press, New York, 1990).
8. I. Talmi, Contemporary Concepts in Physics, Vol. 7: Simple Models of Complex Nuclei (Harwood Academic Publ., 1993).

9. K. Heyde, The Nuclear Shell Model (Springer Verlag, Heidelberg, 1994).

10. I. Talmi, Advances in Nuclear Physics, Vol. 27 (Kluwer Academic Publ., 2003) Chapt. 1: "Fifty Years of the Shell Model - The Quest for the Effective Interaction".

11. I.M. Green, S.A. Moszkowski, Phys. Rev. B 139, 790 (1965).

12. R. Arvieu, S.A. Moszkowski, Phys. Rev. 145, 830 (1966).

13. A. Plastino, R. Arvieu, S.A. Moszkowski, Phys. Rev. 145, 837 (1966).

14. A. Faessler, A. Plastino, S.A. Moszkowski, Phys. Rev. 156, 1064 (1967).

15. J.W. Ehlers, S.A. Moszkowski, Phys. Rev. C 6, 217 (1972).

16. S.A. Moszkowski, Phys. Rev. C 19, 2344 (1979).

17. S.A. Moszkowski, Phys. Rev. C 32, 1063 (1985).

18. M. Moinester, J.P. Schiffer, W.P. Alford, Phys. Rev. 179, 984 (1969).

19. M. Moinester, J.P. Schiffer, W.P. Alford, Phys. Rev. 185, 1598 (1969).

20. J.P. Schiffer, Ann. Phys. 66, 798 (1971).

21. J.P. Schiffer, W.W. True, Rev. Mod. Phys. 48, 191 (1976).

22. L. Coraggio, A. Covello, A. Gargano, N. Itaco, T.T.S. Kuo, D.R. Entem, R. Machleidt, Phys. Rev. C 66, 021303 (2002).

23. C. Mazzocchi, R. Grzywacz, J.C. Batchelder, C.R. Bingham, D. Fong, J.H. Hamilton, J.K. Hwang, M. Karny, W. Krolas, S.N. Liddick, A.F. Lisetskiy, A.C. Morton, P.F. Mantica, W.F. Mueller, K.P. Rykaczewski, M. Steiner, A. Stolz, J.A. Winger, Phys. Lett. B 622, 45 (2005).

24. National Nuclear Data Center, Brookhaven, Evaluated Nuclear Structure Data File, http://ie.lbl.gov/ensdf/. 25. C. Scholl, P. Petkov, V. Werner, A. Linnemann, T. Adachi, A. Dewald, A. Fitzler, C. Fransen, Y. Fujita, J. Jolie, K. Langanke, A.F. Lisetskiy, G. Martinez-Pinedo, D. Mücher, J.N. Orce, N. Pietralla, N. Warr, K.O. Zell, P. von Brentano, Phys. Rev. C 75, 064321 (2007). 